\title{
Implementation of Tensor Flow for Real-time Object Detection
}

\author{
R. Aroul Canessane, R. Dhanalakshmi, V. Maria Anu
}

\begin{abstract}
Tensor Flow is an open-source Machine Learning library for research and creation. Tensor Flow offers APIs for beginners and specialists to create for work desktop, mobile, web, and cloud. The best utilizations of Google's Tensor flow are the best applications for deep learning . Deep Learning is extraordinary at example acknowledgment/machine recognition, and it's being connected to pictures, video, sound, voice, content and time arrangement information. It groups and bunch information like that with now and again superhuman precision. This can be actualized for the acknowledgment of the diverse items, for example, Ball, Cat, Bottle, Car and so forth. It can utilize Android as its stage with to utilize the cell phone's camera to prepare the informational indexes and perceive diverse items in ongoing process.
\end{abstract}

Keywords: Deep learning, Tensor Flow, CNN, Face acknowledgment, Support Vector Machine.

\section{INTRODUCTION}

Numerous issues in PC vision were immersing on their precision before 10 years. Be that as it may, with the increasing of deep learning procedures, precision of this issue definitely will improve. Any serious issues of photo classification that is characterizing of foreseeing the picture. Marginally convoluted issue is picture confinement, this picture containing solitary article for framework ought to foresee the class of the area of the item in the picture (a jumping box around the item). The more entangled issue, of item discovery includes both order and confinement. For this situation, the contribution of framework can be picture, this yield is a jumping square shape relating the every one of these items for this picture, alongside the instance of class container. A review for every one of this issue is shown below.

The notable usage item identification is profile recognition; it can be utilized as practically for any ambulant lens. This progressive summed up request could utilized at independent operating emplacement an assortment items distinguished. Likewise this vital task for carrying out observation frameworks. This framework contain coordinated by different assignments, for example, present guessing the key role in the specified part identify some item, then after that the next scene to evaluate present at recognized area. This project very well utilized in following articles, this type of way utilized at mechanical technology, medicinal

Revised Version Manuscript Received on 10 September, 2019.

R. Aroulcanessane, Sathyabama Institute of Science and Technology, Chennai, Tamilnadu, India

(email: aroulcanessane@gmail.com) R.

R.Dhanalakshmi, Jeppiaar Engineering College, Chennai, Tamilnadu,

V.MariaAnu, Sathyabama Institute of Science and Technology, Chennai, Tamilnadu, India petitions. Along these lines this issue serves a huge number of uses.

\section{Computer Vision Tasks}

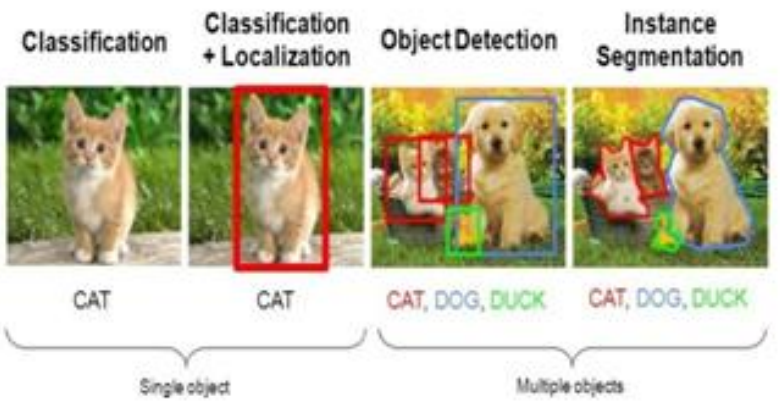

Figure 1: PC Percepfion Chore

The significant testing issue is due to the element of the various articles, they are available on some random info picture. Any broad AI errand wants stable component information and then it ready for detecting the given tasks. The main part is vital obstruction far reaching selecting item discovery frameworks is the necessity of ongoing while at the same time being precise in recognition. Many unpredictable tasks takes a lot of time for derivation then the perplexing task takes less time and gives little exactness. Exchange among exactness and execution related to picked task. This issue includes order just as relapse, driving the model to be adapted all the while. This adds to the unpredictability of the issue.

\section{II.RELATED WORK}

Object recognition is an essential, yet difficult vision assignment[1-9]. It is a basic part in numerous applications, for example, picture look, picture auto-comment and scene understanding, object following. Moving item following of video picture successions was a standout amongst the most essential subjects in PC vision. It had just been connected in numerous PC vision field, for example, savvy video reconnaissance, man-made consciousness, military direction, security identification and robot route, medicinal and organic application (Almeida and Guting, Hsiao-Ping Tsai , Nicolas Papadakis and AurelieBugeau 2017).

In different fields there is a need to identify the objective article and furthermore track them successfully while dealing with impediments and other included complexities. Numerous scientists endeavored for different methodologies

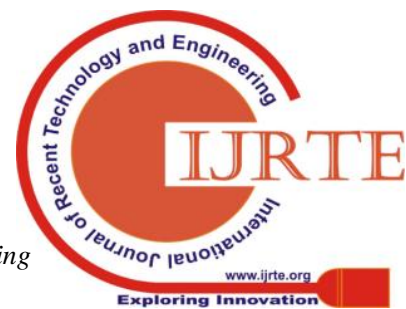


in object discovery. The idea of the strategies to a great extent relies upon the application area (Tie Liu ,2018).

Visual reconnaissance in powerful scenes, particularly for people and vehicles, was a standout amongst the most dynamic research subjects in PC vision. It has a wide scope of potential applications, for example, traffic observation in urban areas, human ID and security monitor for networks or essential structures, and so on. As a rule, the preparing system of visual observation in powerful scenes including the accompanying stages: objects discovery, comprehension and depiction of practices and human distinguishing proof. Conduct understanding and human recognizable proof rely upon the aftereffects of items location which is a standout amongst the most critical parts in visual reconnaissance

( Weiming $\mathrm{Hu}$ et. al ,2018).

\section{III.EXISTING SYSTEM}

Certain applications recognize objects with different characteristics such as shape size etc., But they are not trained to recognize a wide variety of objects. Many applications include only the textual representation of the object in the description but not the voice or an audio effect.

\section{IV.PROPOSED SYSTEM}

This app allows identification of the object but the advantage here is the audio output along with the google talkback (a screen reader software that comes pre installed with any android smartphone). Also, it can be trained to identify the new objects using the tensor flow's efficient algorithm and deep learning .

\section{MODULES \\ Collected Information \\ Generating and train the module \\ - Checking and transport the module}

Installing in android

It needs face identification before face acknowledgment, and the techniques for face recognition incorporate measurable models, Support Vector Machine (SVM) strategy, just as versatile chart coordinating strategy. In measurable component extraction strategies dependent on parameters, the center thought of highlight extraction and highlight point situating is to respect the piece of the picture includes as a sort of model data, and the element of an expansive number of tests and no element tests are prepared (and to prepare an extensive number of highlight class test and non-highlight class test individually), at last extraordinary classifiers are built for highlight extraction and consequent acknowledgment. In this paper, the learning calculation dependent on Adaboost is embraced, just and proficiently.

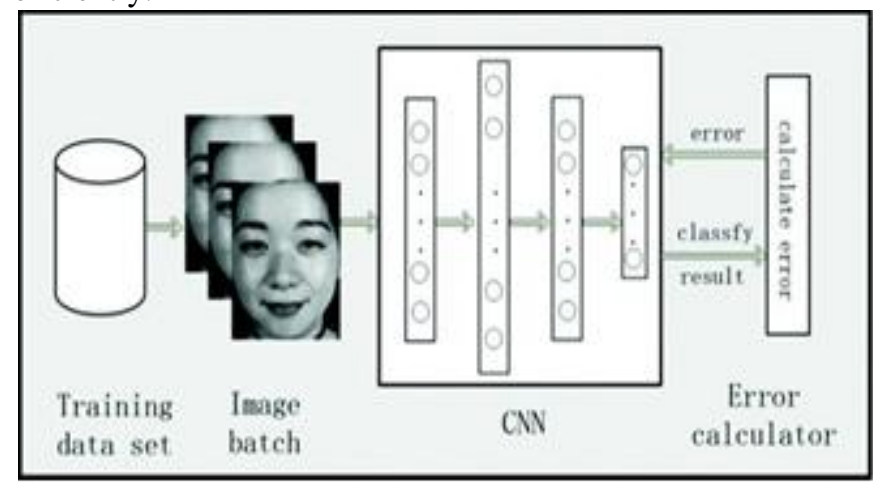

Figure 2: Model of CNN

\section{V.PROGRESS\& RESULTS}

The system utilized in this undertaking depends on Single shot recognition architecture. The design is appeared in below figure

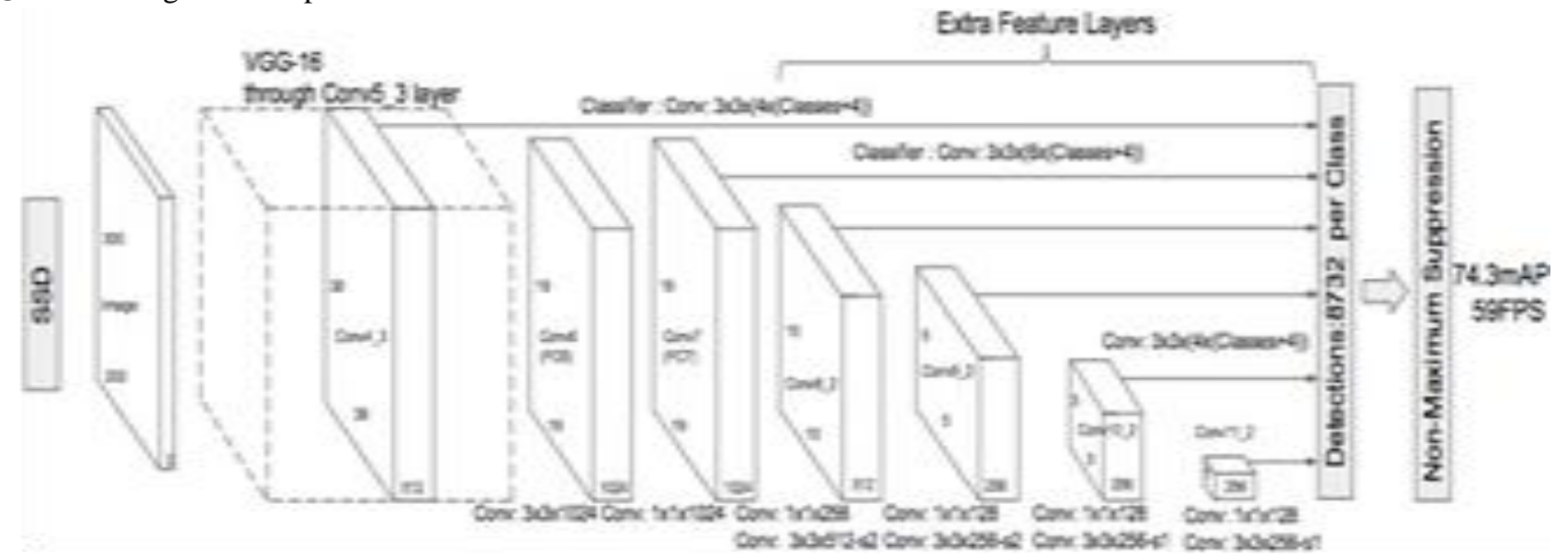

Figure 3: Architecture

The architecture typically begins through display, This type of changing over completely convolutional organize. At that point we join some additional convolutional layers, that assistance to deal with greater items. The yield at the Visual Geometry Group arrange component map. The additional sheet manufacturing, element .Component utilized to foresee jumping square shapes in different scenarios (sheets in charge of bigger articles). In this way the general thought of SSD. A portion in initiations transferred to some part.
Grapples (gathering over square shapes on the picture in various areas, perspective proportions) go about similar focuses in pictures appeared below. This type is prepared for forecasts in every stay remains related to it. 


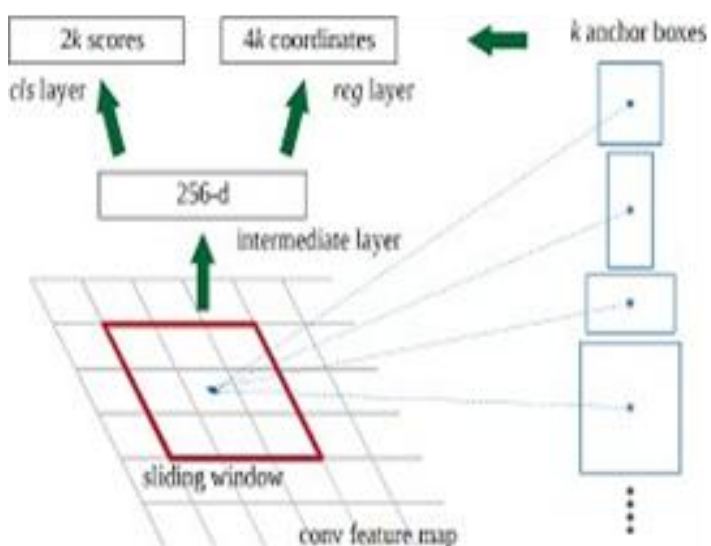

Figure 4: Anchors

Any stay with an IoU (jaccard During getting ready remarks towards catches. Every segment and its components (different partition) are more unmistakable seen as slide. Let us assume showed up below figure, those animals remain composed in canine single hook facilitated. Notice the facilitated in various parts
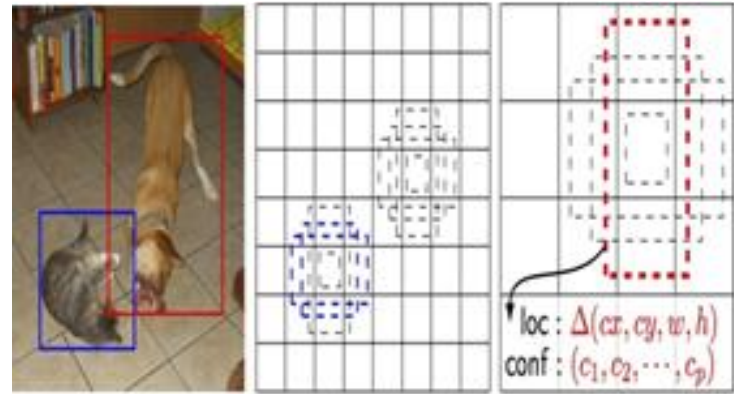

(a) Image with GT bores (b) $8 \times 8$ feature map $\quad$ (c) $4 \times 4$ feature map

Figure 5: Fixtures

This misfortune work utilized is the many squares shape characterization, relapse misfortune. This order misfortune utilized in soft max and relapse smart misfortunes are utilized. Amid expectation, in checking the image concealment utilized for channel different shapes occurred might be coordinated as shown in the below figure.

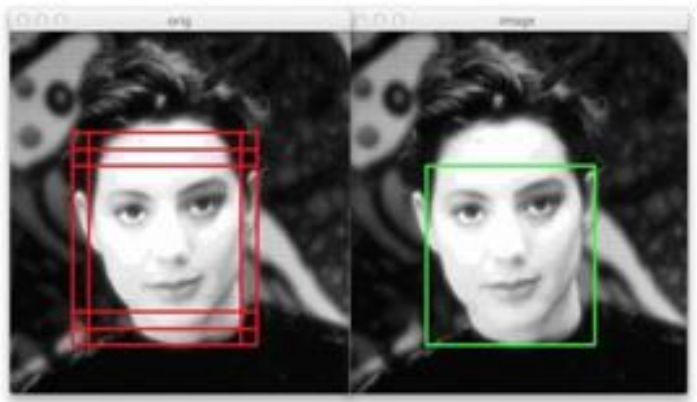

Figure 6: Local Maxima Image

\section{VI.SYSTEM DESIGN}

\section{INFORMATION}

A definitive end goal of the project undertaking, as open source accessible to all users should be utilized. By training the module with different objects in many ways gives the perfect result. If it fails also, we have to train the module in all perspective angles of the picture. Then only the prediction may be possible otherwise it's difficult to predict.

\section{EXECUTION FEATURES}

The undertaking is running in python and utilized in preparing the profound system is utilized in picture predictions. The comment on information is given in XML position, which is perused and put away into a pickle document alongside the pictures with the goal that perusing can be quicker. Likewise, the pictures are resized to a fixed size. The whole system engineering is appeared above. The module comprises system after that it adjusted for calibrating afterward the classifications of systems and localization. It makes a profound system to prepare for start and finish on the particular data.

\section{VII.CONCLUSION}

The execution of face acknowledgment is regularly poor in an obliged domain, for example, enlightenment changes, shooting edge, separation, posture, and appearances, etc which will influence the precision of acknowledgment. Contrasted and conventional hand-created highlights, CNN learning highlights have better power to confront acknowledgment in complex conditions. $\mathrm{CNN}$ is for the most part used to recognize the removal, scaling and different types of bending invariant two-dimensional designs. Since CNN's component recognition layer learns via preparing information, it dodges the element extraction of the showcase and verifiably gains from the preparation information when $\mathrm{CNN}$ is utilized. Additionally, on the grounds that the loads of the neurons on a similar element map are the equivalent, the system can parallel realizing, which is a major preferred standpoint contrasted and the convolutional system of neurons associated network. Tensor Flow is the most recent second-age of Google man-made reasoning learning framework dependent on profound learning. DistBelief system, which has been improved in all angles, better execution, completely open source and can be kept running on more gadgets. In the current face acknowledgment explore, the presentation and utilization of the system is less, and the examination demonstrates that we have accomplished a superior face acknowledgment impact in Tensor Flow structure. In the preparation of $\mathrm{CNN}$, we order the preparation tests as indicated by the posture, and pretreat the preparation information.

\section{REFERENCES}

1. Ross Girshick, Jeff Donahue, Trevor Darrell, and Jitendra Malik. Rich feature hierarchies for accurate object detection and semantic segmentation. In The IEEE Conference on Computer Vision and Pattern Recognition (CVPR), 2014.

2. Ross Girshick. Fast R-CNN. In International Conference on Computer Vision (ICCV), 2015.

3. ShaoqingRen, Kaiming He, Ross Girshick, and Jian Sun Faster R-CNN: Towards realtime object detection with region proposal networks. In Advances in Neural Information Processing Systems (NIPS), 2015. 
4. Joseph Redmon, SantoshDivvala, Ross Girshick, and Ali Farhadi. You only look once: Unified, real-time object detection. In The IEEE Conference on Computer Vision and Pattern Recognition

5. L.MaryGladence,Karthi,M.,Ravi, T "A novel technique for Multi-class ordinal regression-APDC" IndianJournal of Science and Technology Vol.9/ No.10/March 2016/1-5

6. Kamala, V. R., and L. MaryGladence. "An optimal approach for social data analysis in Big Data." In 2015 International Conference on Computation of Power, Energy, Information and Communication (ICCPEIC), pp. 0205-0208. IEEE, 2015.

7. Sneha, SaumyaNath, L.MaryGladence, "Security for Bicycle and Investigation of Health of Bicycle Rider using IOT", in Journal of Advanced Research in Dynamical and Control Systems, 16-Special Issue,2017 Page. No. 1298-1303

8. Brumancia, E., S. Justin Samuel, L. Mary Gladence, and KarunyaRathan. "Hybrid data fusion model for restricted information using Dempster-Shafer and adaptive neuro-fuzzy inference (DSANFI) system." Soft Computing: $1-8$. 